\title{
Transverse Metatarsal Head Resection-A Radical Approach to the Problems of Forefoot Ulceration
}

\author{
JOHS G. ANDERSEN \\ Alupe Leprosy Hospital, P.O. Box No. 35, \\ Busia Market, Kenya
}

\begin{abstract}
Transverse metatarsal head resection is recommended as a surgical approach to the problem of recurrent forefoot ulceration. This operation combines the soundness of a formal foot amputation with due consideration for the desires of patients. Indications and contra-indications are given, and the technique, described in detail, does not require sophisticated orthopaedic experience. The final results are extremely encouraging.
\end{abstract}

Ulceration of the forefoot is a common disability in leprosy patients. For many years it has been an established policy that such conditions should be treated as conservatively as possible.

Even with the best footwear and with the best care many of these patients return time and time again with fresh ulcerations. This is a heavy drain on the economy of the patient, and also on the hospital. There is an evident need for an approach to these problems that gives the patient a better chance of avoiding the disastrous results of recurrent ulceration.

Provided we are dealing with a foot with reasonably good plantar tissue in the mid- and hindfoot, and with reasonably good skeletal alignment of the tarsal region, a formal forefoot amputation is undoubtedly a sound approach. Performed with proper technique and on sound indications this approach reduces the incidence of re-ulceration considerably. The main objection to this is not so much surgical as psychological. Most of our patients are sentimentally quite attached to their toes, even though they may have been reduced to purely ornamental appendages. This we have to accept. We are not going to face life with obvious stigmata of leprosy.

This paper presents a surgical approach to this problem which combines the soundness of the formal forefoot amputation with due consideration for the desires of the patients.

The indications are that any kind of forefoot ulceration or of foref oot scarring poses a serious threat of frequent and progressive ulceration. Contraindications are ulceration and/or scarring of the mid- or hindfoot, since such conditions would not permit safe weightbearing on the reduced foot. Disorganisation and/or malalignment of the tarsal skeleton or the ankle joint are not, as such, contraindications. Such conditions usually require additional, drastic and sophisticated surgery. This, however, is outside the scope of this paper. 

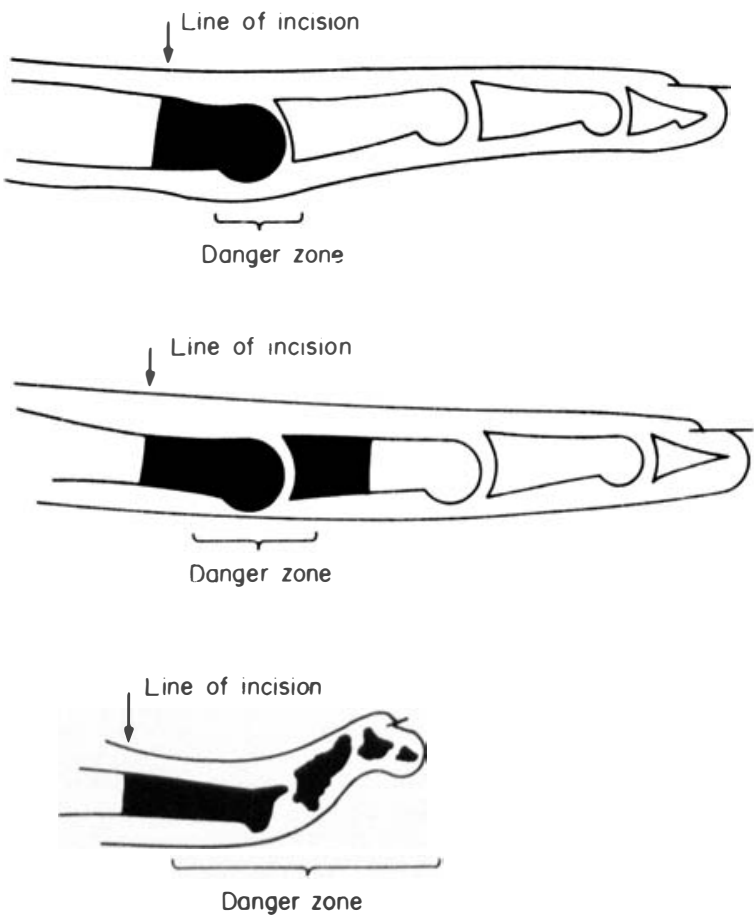

Fig. 1. Diagrammatic representation of the line of incision and of the amount of bone to be removed in three typical operations.

Proper surgical anaesthesia is necessary. Only if the operation is performed in a bloodless field can it be carried out in a satisfactory way.

The operation aims at removing sufficient amounts of skeleton from the forefoot so that the cut ends of the metatarsal bones are well proximal to the proximal edge of the ulcer/scar. This permits a troublefree take-off phase.

The incision is made transversely on the dorsum of the foot at the level of the proposed ostectomy of the metatarsal bones. It should be realised that this level is determined not by any considerations for the anatomy of the foot, but by the necessity of securing a sound take-off pad. The incision is carried down to the level of bone. No attempts at tendon repair are made. The metatarsal bones are divided subperiosteally in a straight transverse line. The distal portions of the bones are twisted out, including the metatarsal heads. Extreme care must be taken to remove any sesamoid bones, and also the occasionally found plate of more or less calcified/ossified osteoid tissue on the plantar surface of the metatarsal bones.

The resulting gap is loosely packed with plain vaseline gauze. The foot is dressed in a bulky dressing. This is left for 3-4 days, or even longer. After this period the foot is treated daily with soaks in plain soapy water and redressed as before. In the few cases where gross discharge of purulent matter or frank necrosis of tissue are found, eusol dressings help in clearing the wound. No indications are recognised for the local application of antibiotics. 
This is essentialiy the regime advocated by the past masters of treatment of osteomyelitis, Trueta, Orr and Chiewitz. The classical instructions call for untouched bandages "until the nurses faint". The recommended technique may not be better, but it does leave the air in the ward fresher and more pleasant.

Soon the cavity starts contracting and granulation tissue appears. Eventually a thin, linear scar is left on the dorsum of the foot. The ulcerated or scarred plantar tissue has been relieved of its adhesions to the skeleton and is allowed to retract. In practically all cases the end result is that the thin, adherent, ulcerprone plantar tissue is replaced by a quite respectable plantar pad.

One variation of this technique calls for removal of the metatarso-phalangeal joints, occasionally even the whole of the proximal phalanges. Usually this is indicated where septic arthritis is a feature, or if simple resection of metatarsal heads and necks does not produce a sufficiently wide gap in the skeleton.

In another variation the distal portions of the metatarsal bones are removed, and all the remnants of bone that are found distal to the former metatarsophalangeal joints are removed. This is frequently indicated where we are dealing with the type of absorbed forefoot, that externally looks rather like a forefoot amputation, but has a very thin, adherent plantar tissue in the take-off area. Wherever indicated this procedure can easily be supplemented by resection of one or more of the proximal interphalangeal joints. It has been found satisfactory to perform the resection exclusively of the fourth and fifth, or of the fif th metatarsal bone, but otherwise following the described technique. This demands good plantar tissue in the remaining forefoot.

A similar approach to the first metatarsal bone is possible, but the results are less satisfactory. The postoperative gait is frequently disturbed.

Isolated metatarsal head resection of one or more of the central metatarsal bones is contraindicated. It does not permit sufficient retraction of the plantar tissue to produce a serviceable plantar pad.

A description of the indications and contraindications for metatarsectomy, removal of one of more metatarsal bones in toto, is outside the scope of this paper. Generally speaking we have found that a short, broad foot-other conditions being equal-is more serviceable and has a higher resistance to re-ulceration than a long, narrow foot.

There are few complications. Very occasionally post-operative bleeding causes anxiety. Proper dressing and properly applied compression bandage, combined with elevation of the operated foot is sufficient to control this. The blood supply to the distal flap may very rarely be insufficient. The result is necrosis of parts of the distal flap. During the $1 \frac{1}{2}$ years this operation has been in regular use in this unit, this has only happened twice. In both cases the end result was a very nice and pleasing forefoot amputation. Pockets of pus may be found tracking proximally in the depths of the foot. They should of course be properly drained, but do not otherwise change the technique or influence the results. Antibiotics are extremely rarely indicated. It is sounder and safer to rely on good surgery rather than on antibiotics.

The final results are extremely encouraging. The dorsal scar usually presents no difficulties. Only occasionally may a deep scar call for secondary plastic revision. The forefoot assumes a normal alignment. The plantar tissue in the ulcer/scar area somehow remodels as a perfectly serviceable take-off pad. We have had only two cases of re-ulceration. In one case a young girl returned with a huge nail perforating the sole of her shoe. In the other case an adult man returned, rather 
shamefaced, to report that he had overlooked a sharp stone in the shoe. In neither case did the re-ulceration occur in the take-off area of the shortened foot.

All have been fitted with protective sandals of the type that routinely are issued to patients with anaesthetic feet. Only if more extensive surgery of the tarsal skeleton has been performed, has more sophisticated footwear been indicated.

In the early days there was a quite understandable reluctance to accept such mutilating surgery. Gradually, as the results became known, the "hidden amputation" as it is known locally, has gained in popularity. It has been interesting to notice that several patients have realised the indications and have requested this operation.

The required instruments, a scalpel, a tissue forceps, a couple of rongeur forceps and a periosteal elevator, are certainly within the reach of any leprosy hospital. The indications and contraindications are clear and do not require sophisticated orthopaedic experience. The technique is simple enough for any physician with some interest in surgery to learn to perform it competently. The results are encouraging enough to warrant use of time, bedspace and money. 